\title{
Encapsulated Sclerosing Peritonitis Successfully Treated with Corticosteroid Therapy and Total Parental Nutrition
}

\author{
Anam Hanut ${ }^{1}$, Rola Nakhoul ${ }^{2}$, Michael Jerdev ${ }^{3}$, Roth Ana ${ }^{1}$, Farid Nakhoul ${ }^{1,4}$, Evgeny Farber ${ }^{1}$ \\ ${ }^{1}$ Nephrology \& Hypertension Division and ${ }^{3}$ Department of Radiology, Baruch Padeh Poriya Medical Center, Tiberias, Israel; \\ ${ }^{2}$ Szeged Faculty of Medicine, Szeged, Hungary; ${ }^{4}$ Azrieli Faculty of Medicine, Ramat Gan, Israel.
}

Corresponding Author:

Dr. Evgeny Farber

Email: Efarber@poria.health.gov.il

This is an Open Access article distributed under the terms of the Creative Commons Attribution License (creativecommons.org/ licenses/by/3.0).

Received : May 8, 2017

Accepted : July 5, 2018

Published : July 25, 2018

\begin{abstract}
Background: The number of patients with end stage renal disease (ESRD) who need renal replacement therapy to survive is increasing worldwide. Continuous ambulatory peritoneal dialysis (CAPD) is the preferred treatment to treat ESRD in an increasing number of patients. However, the long-term effectiveness of CAPD is limited by complications such as peritonitis. Encapsulating sclerosing peritonitis (ESP), a rare cause of bowel obstruction with high morbidity and mortality, was described as a complication associated with peritoneal dialysis. Case Report: Here we report a case where early abdominal $\mathrm{CT}$ in association with clinical symptoms allowed non-invasive diagnosis of ESP, and immediate steroid treatment successfully relieved the symptoms with excellent outcome. Conclusion: Early diagnosis and institution of corticosteroid therapy is effective treatment of encapsulating sclerosing peritonitis secondary to peritoneal dialysis.
\end{abstract}

Keywords: Peritoneal Dialysis, Peritoneal Fibrosis, Intestinal Obstruction, Ultrasonography, Computed Tomography.

\section{Introduction}

Patients with end-stage renal disease (ESRD) have three options of replacement therapy: peritoneal dialysis (PD), hemodialysis (HD), and renal transplant. Continuous ambulatory peritoneal dialysis (CAPD) is a successful dialysis modality in patients with ESRD. This home-based treatment has many advantages by improving their quality of life. In general, survival outcomes of PD are equal to those of HD [1].

Early recognition and treatment of encapsulating sclerosing peritonitis (ESP) secondary to CAPD is essential in the management in order to reduce the high incidence of morbidity and mortality. It is important to be clinically aware of the possibility of ESP presenting many years after stopping peritoneal dialysis [2-4]. ESP usually occurs in patients receiving PD for more than 4 years and the incidence of ESP in patients on PD treatment for less than 2 years is very low [5-7]. ESP presents after withdrawal from PD in the majority of patients $(70-90 \%$ in some series) $[8,9]$. This rare complication is associated with high morbidity and mortality due to bowel obstruction and malnutrition and profound hypo-albuminemia. The reported mortality of this condition is around $50 \%$, usually within 12 months of the diagnosis [10-13].

The most effective treatment of ESP is early diagnosis with immediate corticosteroids at a dose of $1 \mathrm{mg} / \mathrm{kg} /$ day that should be started as soon as possible in early stages. Tamoxifen has also been recommended in severe cases $[5,11]$; however, there are minimal data supporting a positive effect of any of the cited medical treatment options. Surgical treatment is exclusively recommended for patients suffering from intestinal obstruction [6]. 


\section{Case Report}

A 26-year-old Arabic man, with body weight 52 $\mathrm{kg}$, BMI=19, had been maintained on CAPD for one year due to ESRD related to biopsy proven membrano-proliferative glomerulonephritis before his first admission at our center. His first episode of complicated peritonitis by Pseudomonas was treated with intra-peritoneal antibiotics but without improvement in his symptoms, and the Tenckhoff catheter was removed. He was at home without any replacement therapy for several months.

He was admitted to our Department of Internal Medicine at Baruch Padeh Poriya Medical Center, due to abdominal pain with distension, without fever or other symptoms. Physical examinations revealed decreased bowel sounds and diffuse abdominal tenderness. Laboratory results showed hemoglobin of $7 \mathrm{~g} \%$ without leucocytosis, normal amylase blood level, hypo-albuminemia of $2.5 \mathrm{~g} / \mathrm{dL}$, blood urea nitrogen $45 \mathrm{mg} / \mathrm{dL}$, blood creatinine $7.5 \mathrm{mg} / \mathrm{dL}$, and normal liver function tests. His past medical history was sickle cell anemia treated with multiple peak cell transfusion and deferoxamine, recurrent attacks of epilepsy treated with sodium valproate, and hypothyroidism treated with levo-thyroxine $100 \mathrm{mcg}$ daily.

During his first admission for abdominal pain and distension, abdominal ultrasound was performed showing large amounts of bloody fluid in the abdominal cavity. Abdominal computed tomography scan (CT) was performed with contrast [Fig.1a,b], showing diffuse thickening of parietal peritoneum with ascites. $1500 \mathrm{ml}$ of bloody fluid was aspirated from his abdominal cavity under guided ultrasound. Diagnosis of ESP was presumed from the classical clinical and radiological findings. Oral prednisone $40 \mathrm{mg}$ daily was started. Because of uremic symptoms, weight loss, general weakness, and severe malnutrition, chronic hemodialysis was initiated via Permacath daily for two weeks followed by three times a week. Because of symptomatic anemia, three blood units
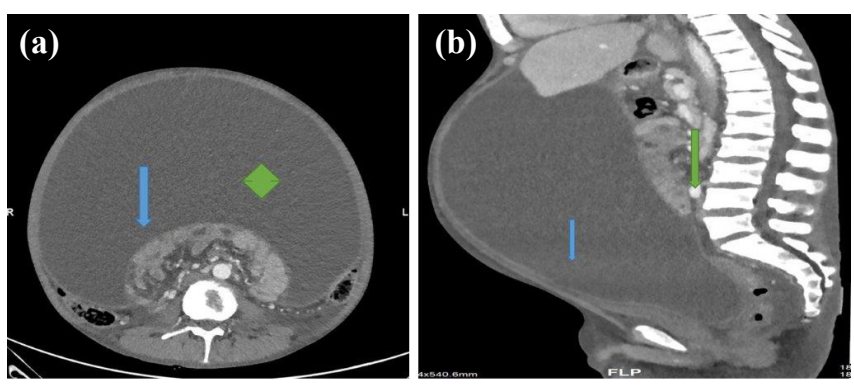

Fig.1: Contrast-enhanced CT scans of the abdomen. Axial (a): and sagittal (b): views show huge amount of ascites, mild diffuse thickening of parietal peritoneum with no significant enhancement and no calcifications, mesenteric thickening with vascular engorgement and thickened and matted appearance of the bowel wall.

were transfused, and intravenous erythropoietin was initiated. Because of recurrent abdominal pain and tension bloody ascites, the patient was admitted different times for paracentesis of ascites. He was readmitted again after four months due to severe cachexia with malnutrition due to loss of appetite, and parenteral nutrition with high caloric index was initiated with every hemodialysis with impressive improvement in his general condition, normalization of his serum albumin, and increased dry body weight.

\section{Discussion}

ESP is classified into idiopathic or secondary. The secondary presentation has been reported mainly in association with CAPD. Peritoneal dialysis is a preferred therapeutic modality that presents several advantages, but with different infectious complications such as bacterial peritonitis. The most severe presentation of this infection is ESP, whose incidence ranges from $0.9 \%$ to $7.3 \%$. The main potential risk factors for development of ESP are the peritoneal dialysis duration and the repeated episodes of peritonitis $[2,3,10]$.

The symptoms are non-specific and present as fever, ascites, weight loss, anorexia, and bowel habit alteration, indicating the presence of an initial inflammatory process. Although 
this is a rare complication, bowel obstruction resulting from ESP should be considered in any patient undergoing long-term peritoneal dialysis with recurrent abdominal pain. As regards the treatment, the therapeutic approach depends on the stage of the disease. Several treatments have been proposed, including pharmacological and surgical. In most cases the surgical treatment is required. Symptoms recurrence occurs in up to $25 \%$ of patients, generally within 12 to 24 months [11].

Candido et al. [3] described a fatal case of sclerosing encapsulating peritonitis in a 43-yearold female patient who had undergone renal transplantation, with immediate post-operative graft failure, when peritoneal dialysis was initiated. Ten years later, the patient was admitted presenting abdominal pain and distension, decrease in dialytic process efficiency, and occasional presence of blood in the dialysis effluent. CT revealed the presence of gross linear calcifications corresponding to thickening and calcification of intestinal and peritoneal walls in association with distended bowel loops with massive ascites in the central region of the abdomen. Later, the patient was admitted presenting vomiting, abdominal distension, and pain, and underwent surgery, progressing with fistulas, hemodynamic instability, and death, with diagnosis of ESP.

Ping et al. [14] reported on five patients who presented to the hospital over a period of 4 years with acute, subacute, and chronic intestinal obstruction. Exploratory laparotomy was performed as there was no resolution of symptoms with conservative treatment. All the patients presented with recurrent episodes (range 1-6) of intestinal obstruction, i.e., colicky abdominal pain, vomiting, abdominal distension, and constipation, over duration of 2 weeks to 10 years prior to attending the hospital emergency department. Contrastenhanced abdomen computed tomography (CT) showed characteristic findings of small bowel loops congregated to the center of the abdomen encased by a soft-tissue density mantle in 4 cases. Exploratory laparotomy was performed for adhesiolysis with excellent recovery.

Acknowledgment: This manuscript was funded by generous contribution of Kraiem Ehmed Magd Elkuroom, North Israel. Contributors: FN, MJ: manuscript writing and literature search; AH, RN, AR: critical inputs into the manuscript, patient management; EF, AR: manuscript editing and patient management. EF will act as guarantor. All authors approved the final version of this manuscript.

Funding: None; Competing interests: None stated.

\section{References}

1. Giannattasio M, Buemi M, Caputo F, Viglino G, Verrina E. Can peritoneal dialysis be used as a longterm therapy for end stage renal disease? Int Urol Nephrol. 2003;35:569-577.

2. Prokesch RW, Schima W, Schober E, Vychytil A, Fabrizii V, Bader TR. Complications of continuous ambulatory peritoneal dialysis: findings on MR peritoneography. AJR Am J Roentgenol. 2000;174:987-991.

3. Candido Pde C, Werner Ade F, Pereira IM, Matos BA, Pfeilsticker RM, Silva R Filho. Sclerosing encapsulating peritonitis: a case report. Radiol Bras. 2015;48:56-58.

4. Stuart S, Booth TC, Cash CJC, Hameeduddin A, Goode JAS, Harvey C, Malhotra A. Complications of continuous ambulatory peritoneal dialysis. Radio Graphics. 2009; 29:441-460.

5. Nakamoto H. Encapsulating peritoneal sclerosis--a clinician's approach to diagnosis and medical treatment. Perit Dial Int. 2005; 25(Suppl 4):S30-38.

6. Carcano G, Rovera F, Boni L, Dionigi G, Uccella L, Dionigi R. Idiopathic sclerosing encapsulating peritonitis: a case report. Chir Ital. 2003;55:605-608.

7. Bargman J, Harel Z. Encapsulating peritoneal sclerosis. US Nephrol. 2011;5:71-75.

8. Afthentopoulos IE, Passadakis P, Oreopoulos DG, Bargman J. Sclerosing peritonitis in continuous ambulatory peritoneal dialysis patients: one center's experience and review of the literature. Adv Ren Replace Ther. 1998;5:157-167.

9. Holland P. Sclerosing encapsulating peritonitis in chronic ambulatory peritoneal dialysis. Clin Radiol. 1990;41:1923.

10. Habib AM, Preston E, Davenport A. Risk factors for developing encapsulating peritoneal sclerosis in the icodextrin era of peritoneal dialysis prescription. Nephrol Dial Transplant. 2010;25:1633-1638.

11. Jung JY, Cho JT. A case of fulminant sclerosing peritonitis presented like acute culture-negative peritonitis and successfully treated with corticosteroid therapy. J Korean Med Sci. 2013;28:620-623.

(C) 2018 Journal of Case Reports
Volume 8, No.3, July-September 2018 
12. Choi JH, Kim JH, Kim JJ, Jin SY, Choi DL. Large bowel obstruction caused by sclerosing peritonitis: contrastenhanced CT findings. Br J Radiol. 2004;77:344-346.

13. Kawanishi H, Moriishi M. Encapsulating peritoneal sclerosis: preventionand treatment. Perit Dial Int.
2007;27(Suppl 2):S289-292.

14. Xu P, Chen LH, Li YM. Idiopathic sclerosing encapsulating peritonitis (or abdominal cocoon): a report of 5 cases. World J Gastroenterol. 2007;13:3649-3651. 